\title{
Documentación
}

CIUDAD Y TERRITORIO

ESTUDIOS TERRITORIALES

ISSN(P): 1133-4762; ISSN(E): 2659-3254

Vol. LII, № 203, primavera 2020

Págs. $171-178$

\section{Crónica de Jornadas del Ministerio de Fomento con motivo de la COP25}

Subdirección General de Políticas Urbanas \& Subdirección General de Suelo, Información y Evaluación Ministerio de Transportes, Movilidad y Agenda Urbana

\begin{abstract}
on motivo de la celebración del $25^{\mathrm{a}}$ Conferencia de las Partes de la Convención Marco de Naciones Unidas sobre el Cambio Climático (COP25), la Dirección General de Arquitectura, Vivienda y Suelo del Ministerio de Fomento, a través de la Subdirección General de Políticas Urbanas y la Subdirección General de Arquitectura y Edificación, ha organizado varias Jornadas que ponían el énfasis en la relación entre el territorio, la ciudad, la arquitectura y la edificación con el cambio climático.
\end{abstract}

\section{Jornada "Agenda Urbana y Cambio Climático"}

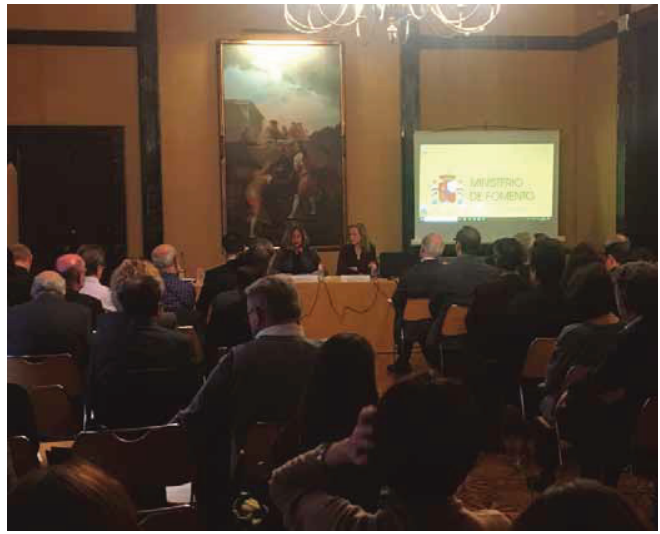

La primera de estas Jornadas se celebró el 4 de diciembre, en el Palacio de Zurbano de Madrid, y con el título "Agenda Urbana y Cambio Climático", tuvo como objeto tratar el papel de las ciudades en relación con la prevención, mitigación y adaptación al cambio climático dentro del marco de la Agenda Urbana Española.

Durante la jornada, que fue inaugurada por la Secretaria General de Vivienda, Helena Beunza, se recordó la vulnerabilidad de las ciudades españolas ante los efectos del cambio climático y cómo la Agenda Urbana y la visión estratégica que propugna suponen una herramienta útil para hacer que nuestras ciudades sean líderes en la lucha contra el cambio climático.

La primera de las dos mesas redondas que conformaron la jornada, fue moderada por la Subdirectora General de Políticas Urbanas, Ángela de la Cruz Mera, y contó con expertos de reconocido prestigio como son Antonio Serrano, Fernando Prats, José Fariña y Carlos Martí, que, respectivamente, analizaron temas tan relevantes como la huella ecológica y la resiliencia urbana, las emergencias climáticas y su relación con la ciudadanía, las ciudades saludables y la comunicación para la sostenibilidad.

La segunda de las mesas, moderada por la Directora de la Función CONAMA, Alicia Torrego, tuvo como objeto la presentación de diferentes 
prácticas llevadas a cabo en el ámbito municipal y que, desde ámbitos diferentes, han afrontado políticas urbanas con repercusiones positivas sobre el medio ambiente y los efectos del cambio climático. Todas las prácticas presentadas fueron acreedoras, en diferentes fechas, de un Premio Internacional de Dubái (concedido por las Naciones Unidas), pero han seguido evolucionando y produciendo efectos favorables hasta el día de hoy.

Entre los ponentes se contó con la participación de Miguel Anxo Fernández Lores, Alcalde Pontevedra, que explicó el "Plan de Movilidad sostenible: una ciudad para las personas"; el responsable del Plan Municipal de Cambio Climático de Noáin (Navarra), Mikel Baztán Carrera; la representante del Observatorio de Medio Ambiente de Málaga, que explicó los trabajos de elaboración del Plan por el Clima: Málaga 2050; la Directora General de Desarrollo Sostenible, Financiación y Acción Exterior del Ayuntamiento de Sevilla, Esperanza Caro, en representación de la Red Española de Ciudades por el Clima y, el Subdirector General de Biodiversidad y Medio Natural del Ministerio de Transición Ecológica, Miguel Aymerich, que expuso la Estrategia de Infraestructura Verde que se está elaborando desde el Ministerio.

En palabras del Secretario de Estado, que clausuró la Jornada que fue un éxito de asistencia, permitió tomar conciencia sobre cómo el cambio climático está afectando a nuestro planeta, a la salud de sus habitantes, a las crisis energéticas y a toda nuestra vida en su conjunto, y sobre e papel que las ciudades representan como actores fundamentales para cumplir, entre otros, e Objetivo estratégico 3 de la Agenda Urbana Española que busca "prevenir y reducir los efectos del cambio climático y mejorar la resiliencia".

\section{Jornada "Arquitectura y Ciudad: claves para un desarrollo sostenible"}

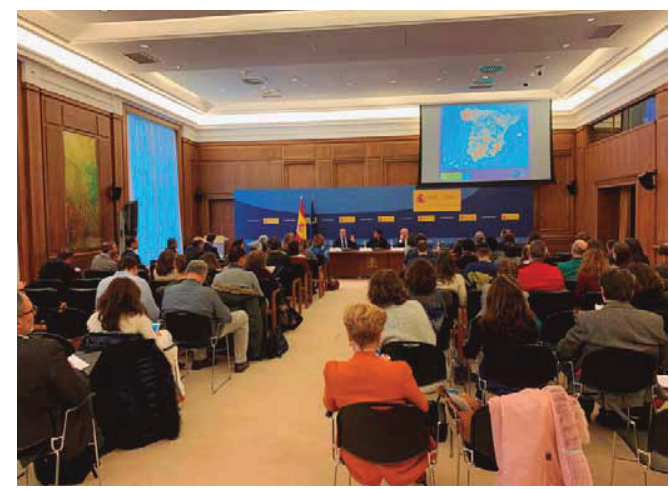

En el mismo marco de la Celebración de la Cumbre del Clima COP25, la Dirección General de Arquitectura, Vivienda y Suelo, en colaboración con Green Building Council España (GBCe) organizó, el 10 de diciembre, en el Palacio Municipal de Congresos de Madrid, la Jornada "Arquitectura y Ciudad: Claves para un desarrollo sostenible".

El objeto principal de esta Jornada fue la presentación de las conclusiones derivadas del Proceso de Participación Pública para la actualización de la Estrategia a largo plazo para la rehabilitación energética en el sector de la edificación en España (ERESEE 2020), que se desarrolló durante el mes de octubre de 2019. Además, simultáneamente a la jornada, y coincidiendo con la segunda parte de la misma, se celebró la segunda reunión del Grupo Interministerial para la ERESEE 2020 con participación de todos los departamentos ministeriales relacionados con la misma.

Tal y como marca la Directiva (UE) 2018/844, la ERESEE tiene como objetivo apoyar la renovación del parque edificatorio español, de edificios residenciales y no residenciales, tanto públicos como privados, para su transformación en un parque inmobiliario con alta eficiencia energética y descarbonizado antes de 2050, facilitando la transformación económicamente rentable de los edificios existentes en edificios de consumo de energía casi nulo.

Para la actualización de esta Estrategia, desde el Ministerio de Fomento se inició un proceso colaborativo de participación pública con distintos representantes de los sectores afectados donde se debatió a lo largo de cuatro jornadas sobre los siguientes temas: la financiación y el modelo de negocio, el vector energético de la rehabilitación, los ayuntamientos y la gestión de la rehabilitación y la comunicación y capacitación del sector. Las conclusiones principales extraídas en cada jornada fueron presentadas respectivamente por Peter Sweatman, Director General de Climate Strategy; Gloria Gómez, Directora General de la Fundación Arquitectura y Sociedad; Marta Rodríguez-Gironés, Subdirectora de Urbanismo de la Federación Española de Municipios y Provincias y Emilio Miguel Mitre, Coordinador internacional de GBCe. Bruno Sauer, Director general de GBCe y Dolores Huerta, Secretaria técnica de GBCe, describieron brevemente el proceso participativo y los principales retos y objetivos del mismo.

Todos ellos, tal y como había adelantado el Director General de Arquitectura, Vivienda y Suelo, Francisco Javier Martín Ramiro, en la inauguración de la Jornada y recogió también el Subdirector General de Arquitectura, Luis Vega, al cierre de la primera parte de la misma, insistieron en la importancia de intervenir en la ciudad conso- 
lidada a través de la rehabilitación y la regeneración urbana poniendo en marcha actuaciones que vayan más lejos que la propia conservación del patrimonio para hacerlo más eficiente energéticamente y más accesible.

Tras el descanso, la segunda parte de la Jornada se diseñó como un foro de reflexión en torno a la rehabilitación que, con la moderación de quien fuera Subdirector General de Innovación y Calidad de la Edificación, Javier Serra, sirvió de marco para exponer los retos de futuro de la edificación desde un punto de vista medioambiental y la regeneración urbana.

Con este objetivo la Subdirectora General de Políticas Urbanas, Ángela de la Cruz Mera, trató la rehabilitación, la regeneración y la renovación urbana en el marco de la Agenda Urbana Española y la Directora del Congreso de Edificios de Energía Casi Nula, Inés Leal, expuso las conclusiones del VI Congreso de Edificios de Energía Casi Nula. A continuación, Emilio Miguel Mitre, presentó el proyecto BUILD UPON 2, un marco de seguimiento de impactos de la rehabilitación energética y la Directora General de Vivienda, Rehabilitación y Regeneración del Área de Gobierno de Desarrollo Urbano del Ayuntamiento de Madrid, Isabel Calzas Hernández, avanzó información relevante sobre el impulso a la rehabilitación energética en la ciudad de Madrid.

Tras la mesa redonda, en la que todo ellos se mostraron de acuerdo en la necesidad de cumplir con las exigencias que nos demanda Europa y sobre la necesidad generar la conciencia sobre la capacidad de la rehabilitación para mejorar la calidad de vida de toda la población, la jornada fue clausurada por el Concejal del Área Delegada de Vivienda, Álvaro González López, y el Director General de Arquitectura, Vivienda y Suelo Fco. Javier Martin Ramiro.

\section{Seminario "Oportunidades para Desarrollo e Innovación en Áreas Rurales"}

La última de estas jornadas celebradas en el marco de la COP25, tuvo lugar el pasado 12 de diciembre en la sede del Ministerio de Fomento. La Dirección General de Arquitectura, Vivienda y Suelo organizó, en colaboración con la red European Urban Knowledge Network (EUKN), el seminario "Oportunidades para el Desarrollo y la Innovación en las Áreas Rurales: Experiencias Europeas / Opportunities for Development and Innovation in Rural Areas: European Experiences".
El seminario se centró fundamentalmente en los desafíos y oportunidades para revitalizar las zonas rurales y los pequeños y medianos municipios frente al fenómeno de la despoblación y se planteó como objetivos clave:

1. Comprender las claves del fenómeno de la despoblación de los núcleos rurales a nivel europeo en función de las experiencias de los países invitados y cómo están afectando el 'bienestar' de los habitantes de dichas áreas.

2. Promover el intercambio de conocimiento, señalando casos de éxito y fracaso de la gobernanza en términos de revitalización rural y

3. Analizar soluciones innovadoras de revitalización de núcleos rurales como objetivos estratégicos para lograr el equilibrio territorial.

Con este planteamiento, el seminario se estructuró en dos sesiones: en la primera de ellas se reunió a expertos que expusieron experiencias interesantes de España, Eslovenia, Alemania, Francia y otros países europeos para abordar la despoblación, fenómeno que se configura como un reto desde múltiples perspectivas y de forma global para el continente europeo.

Como señaló el Director General de Arquitectura, Vivienda y Suelo del Ministerio de Fomento, Francisco Javier Martín Ramiro, en su discurso de apertura, este es un momento crítico para que Europa aborde la despoblación de manera holística, instando a un enfoque más integrado y sistémico de nuestro territorio: es impensable diseñar soluciones si no se tiene en cuenta el continuo rural-urbano. En la misma línea, el Director del Comisionado del Gobierno frente al Reto Demográfico, Ignacio Molina, insistió en esta visión territorial debido a que la despoblación, si bien afecta de forma acusada al medio rural en España, no es exclusiva del mismo y comprende también el medio urbano y la totalidad del territorio.

La sesión además contó con las intervenciones de Mart Grisel, Director de EUKN, que expuso casos de éxito en Alemania, Francia y Letonia; Boštjan Cotič, Director de Proyectos del Instituto de Planificación Urbana de la República de Eslovenia que planteó los desafíos que conlleva la despoblación rural para la planificación espacial en su país; Mireia Sanabria representante de URBACT y José Luis Palma, letrado del Consejo de Estado y Of Counsel en Gómez-Acebo \& Pombo, que abordó las oportunidades para luchar contra la despoblación desde la perspectiva del 
desarrollo e innovación de la agricultura. La sesión culminó con la constitución de una mesa debate entre los ponentes moderada por Mart Grisel.

En la sesión vespertina, se organizaron 5 grupos de trabajo donde todos los participantes en el seminario tuvieron la oportunidad de poner en común sus ideas y aportaciones para lograr el desarrollo, la revitalización y la innovación en las áreas rurales en España. Para concluir la jornada, los moderadores de cada uno de los grupos de trabajo expusieron las conclusiones que alcanzaron en sus respectivos ámbitos:

- Grupo 1. Relación entre infraestructura/servicios físicos y sociales y el ámbito económicoproductivo, moderado por Mart Grisel, Director EUKN.

- Grupo 2. El continuo rural-urbano y la agricultura, moderado por Eulalia Elena Moreno de Acevedo Yagüe, Directora General de Urbanismo y Ordenación del Territorio de Extremadura.
- Grupo 3. Oportunidades para la digitalización de las zonas rurales, moderado por Antonio Alcolea, Subdirector General de Fomento de la Sociedad de la Información.

- Grupo 4. Turismo y tradición para la revitalización del territorio rural, moderado por Luis Ángel Collado Cueto, Profesor de la Universidad Autónoma de Madrid, Facultad de Ciencias Económicas y Empresariales.

- Grupo 5. Cambio climático y el entorno urbanorural, moderado por Amanda del Río, Directora Técnica de la Fundación Global Nature.

En el seno de estos grupos de trabajo se profundizó en los importantes vínculos entre la despoblación y el cambio climático, los sectores económico y productivo (en especial la agricultura), el turismo y la tradición, la innovación digital, así como el marco de referencia de políticas como la Agenda Urbana Española y la Agenda Urbana para la UE. 


\section{Documentación}

\section{Información sobre Tesis doctorales}

\section{Doctoral thesis information}

Título:

"Gobiernos locales y cambio

climático: políticas públicas

e instrumentos jurídicos"

\section{Autora: Doctora Sofia Sımou sofia.simou@uam.es}

Universidad de lectura:

Universidad Autónoma de Madrid

\section{Departamento:}

Derecho público y filosofía jurídica

\section{Programa de doctorado:}

Programa de Doctorado en Derecho, Gobierno y Políticas Públicas por la Universidad Autónoma de Madrid

\section{Fecha de lectura:}

23.09.2019

Calificación:

Doctorado Internacional Sobresaliente Cum Laude

Mención Europeo / Mención Internacional:

Concedido

Director/es de la Tesis y filiación:

Francisco Velasco-Caballero (Catedrático de Derecho Administrativo UAM), Silvia DíEZ-SASTRE (Profesora Contratada Doctora UAM)

\section{Tribunal y filiación de los miembros:}

José María Rodriguez-de SANTIAgo (Catedrático de Derecho Administrativo, UAM). Alba NogueIRALóPEZ (Catedrática de Derecho Administrativo, Universidad Santiago de Compostela). Martin BuRgI (Profesor Catedrático, Universidad de Munich Ludwig-Maximilians-Universität München)

\author{
Enlace a documento de la Tesis completo en \\ repositorio de la Universidad o Ficha Teseo: \\ https://www.educacion.es/teseo/ \\ mostrarRef.do?ref=1816626
}

\section{Resumen:}

La tesis doctoral con el título "Gobiernos locales y cambio climático: políticas públicas e instrumentos jurídicos" tiene como objeto determinar el margen de actuación político-jurídica de los gobiernos locales en España en materia de cambio climático a la luz de las bases y límites constitucionales y legales actuales. A lo largo de la tesis doctoral se realizan referencias a otro tipo de factores de naturaleza organizativa, económica y práctica que pueden condicionar el margen local. El estudio se basa en la hipótesis de que los gobiernos locales en sus tres posiciones elementales (como reguladores -normativos y controladores-, agentes económicos activos en el mercado-proveedores de servicios, partes contratantes, administradores de su propio patrimonio, operadores en el contexto la iniciativa económica pública-y promotores de políticas públicas, -incentivando o desincentivando ciertas actuaciones-) ostentan un espacio de actuación suficiente que les permite diseñar y juridificar sus propias políticas de cambio climático relacionadas tanto con la mitigación como con la adaptación al fenómeno físico. En este sentido, en la parte especial de la tesis se ha llevado a cabo una investigación transversal entre las cuatro áreas materiales más incidentes de la actividad administrativa local sobre la aceleración del cambio climático (planificación urbanística, energía, movilidad y gestión de residuos). A partir del material sectorial se ha construido también la parte general de la tesis que recoge de forma abstracta y horizontal los instrumentos jurídicos locales existentes en esta materia y, cuando necesario, propone las formas y límites para su conversión en instrumentos jurídicos "filoclimáticos". 
Título:

\section{"La anulación de los instrumentos de planeamiento urbanístico"}

Autora: María José VALenzuela-Rodríguez maria.jose.valenzuela.rodriguez@xunta.gal

Universidad de lectura:

Universidad de Vigo

\section{Departamento:}

Derecho público y filosofía jurídica

\section{Programa de doctorado:}

Programa de Doctorado Ordenación Xurídica do Mercado por la Universidad de Vigo

\section{Fecha de lectura:}

30.01.2019

\section{Calificación:}

Sobresaliente Cum Laude

Director/es de la Tesis y filiación:

Dra. Patricia Valcárcel Fernández (Universidad de Vigo)

Tribunal y filiación de los miembros:

presidido por D. Ángel MENÉNDEZ-REXACH (Universidad Autónoma de Madrid) y del que formaron parte también la Dra. Judith GIFREU I FONT (Universidad Autónoma de Barcelona) y el Dr. Tomás Alberto QUINTANA-LóPEZ (Universidad de León)

Enlace a documento de la Tesis completo en repositorio de la Universidad o Ficha Teseo:

https://www.educacion.gob.es/teseo/mostrarRef.do?ref $=1726677$

\section{Resumen:}

El plan urbanístico es el instrumento llamado a diseñar el modelo de ocupación del suelo, los usos posibles y su distribución o interrelación, la ubicación de equipamientos y zonas de esparcimiento, las ordenanzas y normas a aplicar, determinando aspectos concretos de la edificación (alturas, tipología edificatoria, parcela mínima, ocupación, etc.) y concretando el contenido del derecho de propiedad de los titulares de los terrenos que se encuentran en su ámbito territorial. Por todo ello, el plan urbanístico se configura como un elemento fundamental para el desarrollo del modelo de ciudad.

La anulación de instrumentos de planeamiento urbanístico se ha convertido en la actualidad en uno de los graves problemas a los que se enfrenta nuestro ordenamiento jurídico. Esta problemática es patente, tanto desde la perspectiva de la relevancia de las consecuencias que se desprenden de tal declaración de nulidad, como desde la óptica cuantitativa, dado el incremento exponencial de planes urbanísticos que han sido objeto de anulación por los órganos jurisdiccionales.

A través de este trabajo de investigación se aborda el régimen legal de los instrumentos de planeamiento urbanístico y se estudian las principales causas que han apreciado los tribunales como determinantes para decretar su nulidad, analizando las consecuencias que de ello se derivan.

Esta situación ha provocado multitud de reacciones e incluso ha reavivado debates en torno a instituciones clásicas del Derecho, como la teoría de la invalidez de los reglamentos. Por todo ello, la doctrina no ha permanecido ajena a esta materia, generando un amplio compendio de propuestas que se han sistematizado en el presente estudio. 


\section{Documentación}

\section{Evaluadores 2019}

\section{Experts 2019}

Los evaluadores del año 2019 de la revista CyTET han sido los siguientes:

\author{
Alba Nogueira-López \\ Alejandro Vergara-Blanco \\ Almudena de Benito-Alonso \\ Andrés González-Sanfiel \\ Ángel Aparicio-Mourelo \\ Ángel Francisco Martín-Ramos \\ Ángela de la Cruz-Mera \\ Ángela de Meer Lecha-Mazo \\ Anna Ma Riba-Palom \\ Antonio Campesino-Fernández \\ Antonio Piñero-Valverde \\ Arlinda García-Coll \\ Benjamín Galacho \\ Blanca Lozano-Cutanda \\ Carles Llop-Torne \\ Carlos Martín-Cantarino \\ Carlos Muñoz-Parra \\ Carolina Yacamán-Ochoa \\ David Cabrera-Manzano \\ Dionisio Fernández de Gatta-Sánchez \\ Dolores Sánchez-Aguilera \\ Eduardo de Santiago-Rodríguez \\ Elia Canosa-Zamora \\ Enrique J. Burban-Valencia \\ Federico López-Silvestre \\ Felipe Fernández-García \\ Fernando Díaz-Orueta \\ Fernando Vera-Rebollo \\ Flor Arias-Aparicio \\ Francisco Javier Jover-Martí \\ Gerardo Roger Fernández-Fernández \\ Ignacio Bisba-Grandal \\ Ignacio Oteiz-San José
}

\author{
Universidade de Santiago de Compostela \\ Universidad Católica de Chile \\ Universidad Rey Juan Carlos \\ Universidad de La Laguna \\ Universidad Politécnica de Madrid \\ Universidad Politécnica de Cataluña \\ S.G. Políticas Urbanas, Ministerio de Fomento \\ Universidad de Cantabria \\ Universitat de Girona \\ Universidad de Extremadura \\ Universidad de Sevilla \\ Universitat de Barcelona \\ Universidad de Málaga \\ Gómez-\& Pombo Abogados, S. L. P. \\ Universitat Politecnica de Catalunya \\ Universidad de Alicante \\ Universidad de Santiago de Chile \\ Heliconia S. Coop. Mad. \\ Universidad de Granada \\ Universidad de Salamanca \\ Universitat de Barcelona \\ S.G. Políticas Urbanas, Ministerio de Fomento \\ Universidad Autónoma de Madrid \\ Universidad de San Buenaventura, Cali - Colombia \\ Universidad de Santiago de Compostela \\ Universidad de Oviedo \\ Universidad de La Rioja \\ Universitat d'Alacant \\ Universidad de Extremadura \\ Universidad de Castilla La Mancha \\ PAU Urbanismo, S.L.P. \\ Universidad de Concepción-Chile \\ Instituto de Ciencias de la Construcción \\ -Eduardo Torroja - CSIC
}


Inmaculada Caravaca-Barroso

Isabel Madurga -Chornet

Isabel Rodríguez-Chumillas

Itxaro Latasa-Itxaro

Jesús Santiago-Ramos

Jesús Varga-Molina

José Carlos Salcedo-Hernández

José Luis Blasco-Díaz

José Vicente Sánchez-Cabrera

Josep Ivars-Baidal

Josep $\mathrm{M}^{\mathrm{a}}$ Aguirre i Font

Juan Cristaldo

Juan José Díez-Sánchez

Juan Miguel Albertos-Puebla

Leandro del Moral-Ituarte

Lluís Frago Clols

Lorenzo Abad-Casal

Lorenzo Mellado-Ruiz

Ma Cruz Porcal-Gonzalo

$M^{a}$ del Pilar Bensunsan-Martín

$M^{a}$ Jesús Vida-Domínguez

Ma Luisa Gómez-Moreno

Maite Verdú-Martínez

Manuel de la Calle-Vaquero

Manuel Valenzuela-Rubio

Marcela Ramón-García

Marcos Ros-Sempere

Marcos Vaquer-Caballería

María Cristina García-González

María Zambonino-Pulito

Mario Torres-Jofré

Marta Domínguez-Pérez

Marta Lora-Tamayo

Miguel Gómez-Villarino

Mireia Baylina-Ferré

Miriam García-García

Oliver Meseguer-Ruiz

Omar Bouazza-Ariño

Pilar Garrido-Sánchez

Santiago Fernández-Muñoz

Silvia Fernández-Cacho

Sonia Hernández-Partal

Tomás Mazón-Martínez

Víctor Fernández-Salinas

Voltaire Alvarado

Xavier Sabaté-Rotés
Universidad de Sevilla

S.G. Políticas Urbanas, Ministerio de Fomento

Universidad Autónoma de Madrid

Universidad del País Vasco/Euskal Herriko Unibertsitatea

Universidad Pablo Olavide

Universidad Pablo de Olavide

Universidad de Extremadura

Universitat Jaume I

Universitat de Valencia

Universidad de Alicante

Universitat de Girona

CIDI FADA de Asunción - Paraguay

Universitat d'Alacant

Universitat de Valencia

Universidad de Sevilla

Universitat de Barcelona

Universidad de Alicante

Universidad de Almería

Universidad del País Vasco

Universidad de Granada

Universidad Autónoma de Madrid

Universidad de Málaga

S.G. Políticas Urbanas, Ministerio de Fomento

Universidad Complutense de Madrid

Universidad Autónoma de Madrid

Universidad de Alcalá de Henares

Universidad Politécnica de Cartagena

Universidad Carlos III de Madrid

Universidad Politécnica de Madrid

Universidad de Cádiz

Universidad de Chile

Universidad Complutense de Madrid

Universidad Nacional de Educación a Distancia

Arquitecto

Universitat Autònoma de Barcelona

LANDLAB Laboratorio de Paisajes

Universidad de Tarapacá - Chile

Universidad Complutense de Madrid

S.G. Políticas Urbanas, Ministerio de Fomento

Universidad Carlos III de Madrid

Instituto de Patrimonio Histórico de Andalucía

S.G. Políticas Urbanas, Ministerio de Fomento

Universidad de Alicante

Universidad de Sevilla

Pontificia Universidad Católica de Chile

Espai TRES Territori i Responsabilitat Social

Nuestro más sincero agradecimiento a la valiosa y desinteresada colaboración de todos ellos que, con sus comentarios y sugerencias, han contribuido a mejorar y enriquecer los manuscritos originales. 